\title{
Low-profile Visualized Intraluminal Support device (LVIS Jr) as a novel tool in the treatment of wide-necked intracranial aneurysms: initial experience in 32 cases
}

\author{
Daniel Behme, ${ }_{1}^{1}$ Anushe Weber, ${ }_{1}^{1}$ Annika Kowoll, ${ }^{1}$ Ansgar Berlis, ${ }^{2}$ Thomas H Burke, ${ }^{3}$ \\ Werner Weber ${ }^{1,4}$
}

${ }^{1}$ Department of Radiology and Neuroradiology, Klinikum-Vest, Knappschaftskrankenhaus, Recklinghausen, Germany ${ }^{2}$ Department of Radiology and Neuroradiology, Klinikum Augsburg, Augsburg, Germany ${ }^{3}$ Microvention-Terumo, Tustin, California, USA

${ }^{4}$ Ruhr-University-Bochum, University Medical Center Knappschaftskrankenhaus Langendreer, Bochum, Germany

\section{Correspondence to} Dr Anushe Weber, Department of Radiology and Neuroradiology, Klinikum Vest-Knappschaftskrankenhaus Recklinghausen, Dorstener Straße 151, Recklinghausen 45657, Germany; Anushe.Weber@Klinikum-Vest.de

AW and WW share the affiliation of the corresponding author.

\section{ABSTRACT \\ Background and purpose The focus of this study was to determine ease of deployment, safety and effectiveness of the LVIS Jr device.}

Methods A retrospective analysis was performed of 32 cases comprising 34 aneurysms in which the LVIS Jr device was used for stent-assisted coil embolization of intracranial aneurysms from February to October 2012, including all clinical and angiographic data as well as mid-term follow-up (1-12 months of treatment).

Results The median age of the patients was 54 years (range 21-76) and 19 (59\%) were women. The aneurysms were ruptured in 12/34 cases (35.3\%); 26 $(76.4 \%)$ were located within the anterior circulation and the remaining $8(23.5 \%)$ were located in the posterior circulation. Eleven of the 34 aneurysms (32.3\%) were treated with a Y-stent configuration. Immediate total occlusion was observed in $16 / 34(47 \%)$, near total occlusion (90-95\%) in 5/34 (14.7\%) and a 'dog ear' or subtotal occlusion in 12/34 (35.2\%). A single aneurysm was treated without coil embolization. Complications occurred in $5 / 34$ cases $(15 \%)$, including two cases of in-stent thrombosis.

Conclusions Implantation of the LVIS Jr device as a support device for stent-assisted coil embolization seems to be safe and effective. The LVIS Jr device can also be implanted in a Y-stent configuration, offering a novel technique with a potentially lower risk of thromboembolic complications compared with other devices.

\section{INTRODUCTION}

Over the last decades, endovascular treatment of intracranial aneurysms has become a commonly used technique. ${ }^{1} 2$ The treatment of wide-necked and complex aneurysms is still challenging. Many devices have been developed to support coil embolization of wide-necked aneurysms - for example, balloon remodeling where a non-detachable balloon is placed along the aneurysm neck/parent vessel interface to bridge the neck of the aneurysm to prevent coil herniation. ${ }^{3-5}$ An alternative technique to protect the inflow zone is the placement of an intraluminal metallic stent bridging the neck to prevent coil herniation or prolapse into the parent vessel. ${ }^{6-12}$ Among others, Spiotta et al ${ }^{13}$ demonstrated the usefulness of multiple techniques using stents and balloons. Both techniques which use balloons or intraluminal stents are very useful tools in maintaining coil stability within the aneurysm sac for sidewall lesions. However, bifurcation aneurysms represent an important subgroup of cases where stent/balloon-assisted coil embolization is technically challenging. The use of the so-called Y-stent configuration has been used in clinical practice and seems to be a promising technique. ${ }^{13-18}$ Using this $\mathrm{Y}$ configuration will create a new 'pseudo-bifurcation point' which changes the inflow patterns into the aneurysm sac. This anatomical change in the vessel orientation could promote an increase in thrombus formation within the coiled aneurysm sac and might therefore promote long-term occlusion of the aneurysm.

Although the use of intraluminal coil-assist stent devices with the intention of coil protection has increased over the years, these devices have been shown to have a secondary purpose. Many authors ${ }^{15} 16$ have advocated that the deployed intraluminal device produces a scaffolding or substrate for neointimal growth which leads to the remodeling of the parent vessel along the aneurysm neck. This neointimal growth along the parent vessel interface with the aneurysm neck isolates the sac from the circulation.

The most widely used stents for coil-assist treatment are the Neuroform system ${ }^{8} 9$ (Stryker, Kalamazoo, Michigan, USA), the Enterprise Vascular Reconstruction Device ${ }^{17-21}$ (Codman Neurovascular, Raynham, Massachusetts, USA) and the Leo stent device $^{22-24}$ (Balt, France). These stents are selfexpanding Nitinol stents. The Neuroform stent is a segmental open-cell design whereas the Enterprise is a closed-cell design. The Enterprise and the Leo stents are partially retrievable prior to deployment and implantation but the Neuroform stent is non-retrievable.

The primary device used in this study is the new Low-profile Visualized Intraluminal Support device (LVIS Jr; MicroVention-Terumo, Tustin California, USA) which is a self-expanding Nitinol single wire braid device with $1.5 \mathrm{~mm}$ cells with three radiopaque tantalum threads within the body of the stent and radiopaque markers on the proximal and distal ends (figure 1). The key feature of the LVIS $\mathrm{Jr}$ device is that it can be deployed through a 0.017 inch luminal microcatheter which can also be used for coil deployment. The LVIS Jr device is 


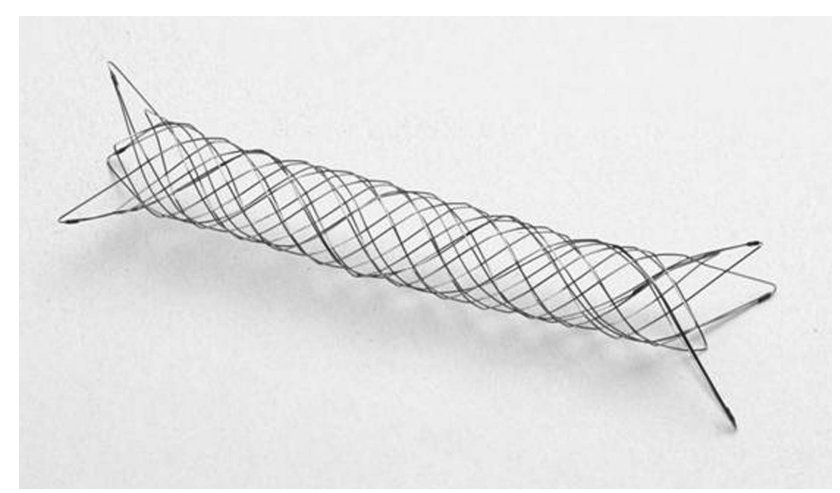

Figure 1 The LVIS Jr coil assist device.

intended for a parent vessel size of $2.0-3.5 \mathrm{~mm}$ as the fully expanded diameter of the device is $3.5 \mathrm{~mm}$; it provides $12-21 \%$ surface area coverage. The LVIS Jr has a CE Mark for use in the EU but it is still under an investigational study protocol in the USA.

\section{MATERIAL AND METHODS}

This was a single-arm retrospective study investigating the safety and effectiveness of the LVIS Jr device for the treatment of wide-necked complex intracranial aneurysms. Two study centers enrolled 32 patients with a total number of 34 aneurysms over a 9-month period from February to October 2012. The patients comprised 19 women (59\%) and 13 men (41\%) of median age 54 years (range 21-76). Twenty-six (76.4\%) of the 34 aneurysms were in the anterior circulation and eight $(23.5 \%)$ were in the posterior circulation; 12 of the aneurysms (35.3\%) were ruptured. The median dome size was $7 \mathrm{~mm}$ (range $2-18 \mathrm{~mm}$ ) and the median neck size was $4 \mathrm{~mm}$ (range 1.5-10 $\mathrm{mm}$ ).

The parent target vessels ranged from $2.2 \mathrm{~mm}(1.1-3.4 \mathrm{~mm})$ distally and to $2.55 \mathrm{~mm}(1.5-5 \mathrm{~mm})$ proximally. Twenty-one aneurysms $(61.7 \%)$ were treated with a single LVIS Jr device and 11 patients $(32.3 \%)$ were treated by Y-stenting: five patients receiving two LVIS Jr stents and six patients were treated by Y-stenting with other previously deployed stents (3 Neuroform, 2 Enterprise and 1 LVIS stent). The primary microcatheter used for deployment of the LVIS Jr stent was the Headway 17 microcatheter (MicroVention-Terumo), which has an inner lumen diameter of 0.017 inch. The microcatheters used for coiling were Headway 17 and Excelsior SL-10 (Boston Scientific; inner diameter 0.0165 inch). Unlike laser cut stents where the stent cells are fixed, the LVIS Jr stent is a single wire closed-cell design with compliant $1.5 \mathrm{~mm}$ cell size. With this braided technology the stent cells are moveable, allowing microcatheters from $0.017 \mathrm{Fr}$ up to $2.0 \mathrm{Fr}$ or a second stent in the Y configuration to be easily recatheterized through the stent cells.

Coil embolization of the aneurysms was either carried out using a jailing technique or through the interstices of the LVIS Jr stent using platinum coils (Microplex 10 Hypersoft Helical and Microplex 10 Helical soft, Microplex 10 Cosmos, Microplex VFC, Microvention/Terumo; or Target 360 standard, Target 360 soft, Target 360 Ultra and Target Helical ultra, Boston Scientific).

The aneurysms were subjectively coil embolized until complete occlusion was achieved or no further coils could be safely deployed within the aneurysm sac. All patients with incidental aneurysms received dual antithrombotic medication with aspirin $100 \mathrm{mg}$ and clopidogrel $75 \mathrm{mg}$ before treatment. Testing for clopidogrel resistance was performed using Multiplate-Analyzer (Roche, Basel, Switzerland). If testing was insufficient for ASPI and ADP, the patient received clopidogrel $4 \times 75 \mathrm{mg}$ and the Multiplate analysis was repeated. In cases of acute subarachnoid hemorrhage, anticoagulation was ensured by periprocedural intravenous administration of aspirin or eptifibatide (Integrilin) after stent deployment.

\section{RESULTS}

All LVIS Jr devices were successfully deployed and implanted without any technical complications. The immediate posttreatment angiograms showed that $17 / 34$ aneurysms (50\%) had immediate complete occlusion of the target aneurysms, 5 (14.7\%) had 90-95\% occlusion, 4 (11.8\%) had subtotal occlusion and $8(23.5 \%)$ had a 'dog ear' occlusion. The follow-up imaging ranged from 1 to 12 months (mean 4.4 months). One patient was lost to follow-up. Of the aneurysms that were completely occluded on the immediate post-treatment angiogram, 94\% remained occluded; one aneurysm that was completely occluded initially converted to residual aneurysm filling. Of the aneurysms that were $90-95 \%$ (nearly total) occluded or small 'dog ear' occlusion initially, 54\% had converted to $100 \%$ occlusion on follow-up imaging. However, the remaining $16 \%$ of aneurysms were either stable or had only a slight increase in filling of the aneurysm sac. These patients will have continued follow-up imaging and, if clinically significant or if the inflow changes from the initial baseline angiogram, retreatment will be considered.

In a subgroup analysis, Y-stenting led to an immediate total occlusion in $9 / 11$ cases $(82 \%)$ and nearly total occlusion (90$95 \%)$ was observed in $2 / 11$ cases $(18 \%)$.

There were five complications associated with the treatment. One patient developed cerebral vasospasm after stent deployment; however, this patient initially presented with an acutely ruptured basilar tip aneurysm with a subarachnoid hemorrhage Hunt and Hess grade I. Two patients presented with thrombus formation after stent placement (figure 2). Both patients were treated with continuous intravenous administration of eptifibatide for $24 \mathrm{~h}$; neither had clinical symptoms and both were asymptomatic. Control digital subtraction angiography (DSA) investigations the following day showed that the thrombus had completely resolved in both cases. Both patients were tested for clopidogrel resistance before treatment but were found to have an adequate response to clopidogrel. One patient who had received stent-supported coiling of a middle cerebral artery (MCA) aneurysm refused further control angiograms. However, 5 months after initial treatment this patient was admitted with an acute MCA hemispheric stroke. DSA revealed in-stent thrombosis; however, the patient had stopped antithrombotic medication. The patient was reloaded with aspirin and clopidogrel but refused further angiographic controls. Finally, one patient had suffered multiple cardioembolic strokes since the initial aneurysm treatment. Due to the poor clinical condition, a control DSA was performed 1 year after the treatment which demonstrated occlusion of the LVIS Jr stent in the A2 segment. Clinically, the patient was symptomatic with a temporary contralateral leg paresis.

\section{DISCUSSION}

The LVIS and LVIS Jr devices have been successfully used in previous reports 25 in the treatment of dissecting aneurysms ${ }^{25}$ and also in a case where the LVIS Jr was successfully deployed after balloon remodeling, with deployment of the LVIS Jr device through the Scepter C balloon (MicroVention-Terumo). ${ }^{26}$ 


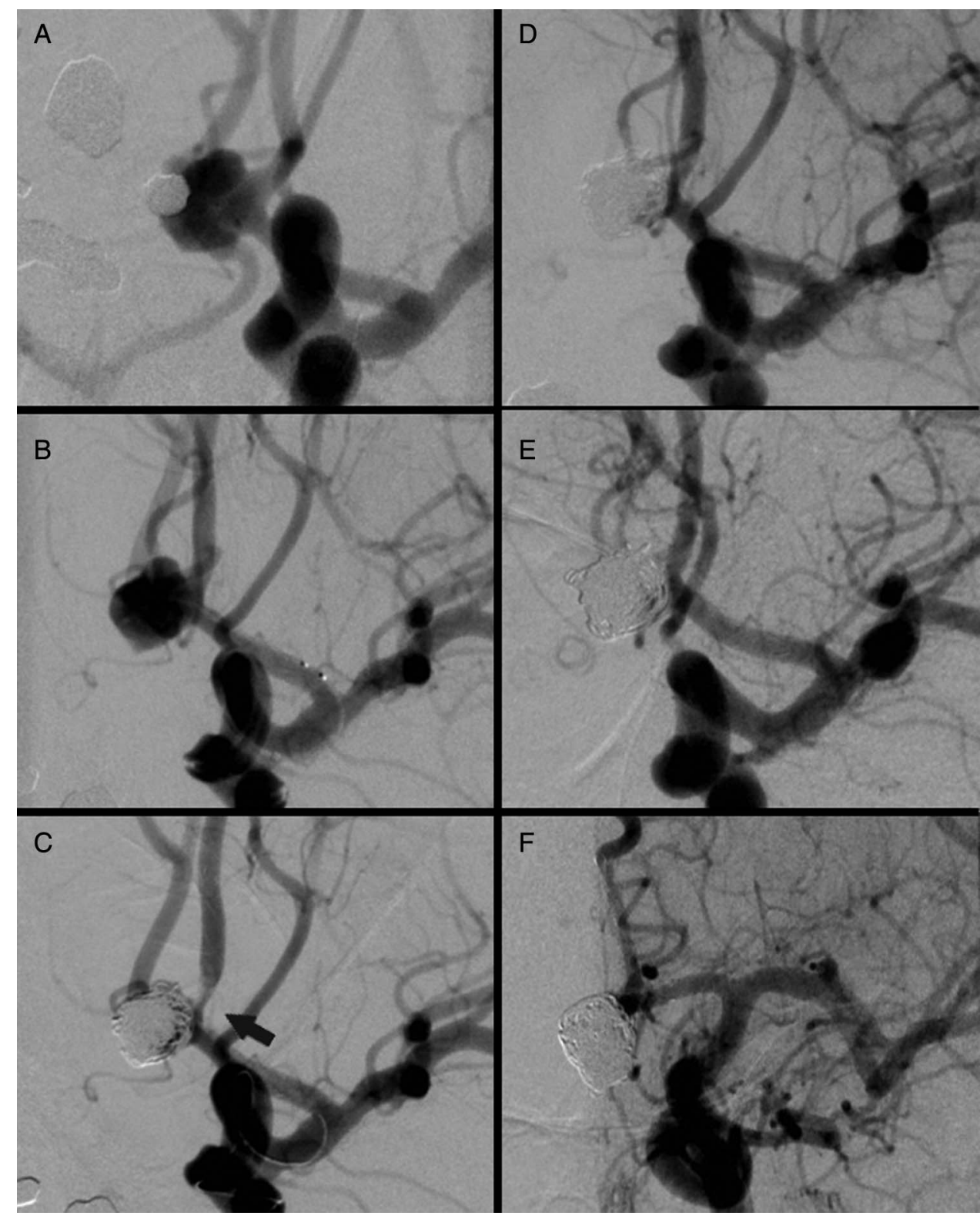

Figure 2 (A) Incidental anterior communicating artery aneurysm. (B) After deployment of the LVIS Jr stent. (C) Control after coil embolization showing formation of a thrombus within the A2 segment of the left anterior carotid artery. (D) After $2 \mathrm{~h}$ intravenous administration of eptifibatide. (E) Control after $24 \mathrm{~h}$ showing that the thrombus had resolved completely. (F) Three-month follow-up showing complete occlusion of the aneurysm.

However, this is the first German experience and initial clinical series using the LVIS Jr device for different types of aneurysm morphologies in ruptured and unruptured aneurysms. In our series the LVIS Jr device was successfully deployed in all cases whereas deployment rates of $80-94 \%$ have been reported when the Enterprise or Neuroform stents were used. ${ }^{27}$ We showed that the use of the LVIS Jr device for stent-assisted coil embolization of wide-necked cerebral aneurysms leads to comparatively high immediate as well as very high mid-term occlusion rates (90\% total, 'dog ear' or near total occlusion). Other groups have found immediate occlusion rates ranging from $46 \%$ to $73 \%$ for total or near total aneurysm occlusion when the Neuroform stent was used for stent-assisted coil embolization $^{8} 927$ and from $76 \%$ to $87.3 \%$ when the Enterprise stent was used. ${ }^{1720} 27$ Similar results have been published for the Leo stent. $^{23}{ }^{24}$ In the subgroup with Y-stenting (figure 3) we found an immediate total occlusion rate of $82 \%$, which corresponds to that described in the literature. A recent multicenter study focusing on aneurysm occlusion after Y-stenting reported that about $83 \%$ of cases achieved immediate total or near total occlusion. ${ }^{18}$ However, these findings are limited by the small number of cases in our series and have to be proved in future trials.

Although the LVIS Jr device is only recommended for a parent vessel size to a maximum of $3.5 \mathrm{~mm}$, one patient in our series had a maximum parent vessel diameter of $5.0 \mathrm{~mm}$ at the proximal landing zone in which the LVIS Jr device was used. The LVIS Jr device was intentionally foreshortened to increase the diameter size to accommodate the parent vessel size. The LVIS Jr device fully apposed to the parent vertebral artery wall well, with complete occlusion of the target aneurysm on immediate and follow-up imaging. This is a potential benefit of a braided stent compared with a laser cut stent.

Subtotal occlusion or a 'dog ear' configuration was not associated with specific target vessel features such as diameter, 


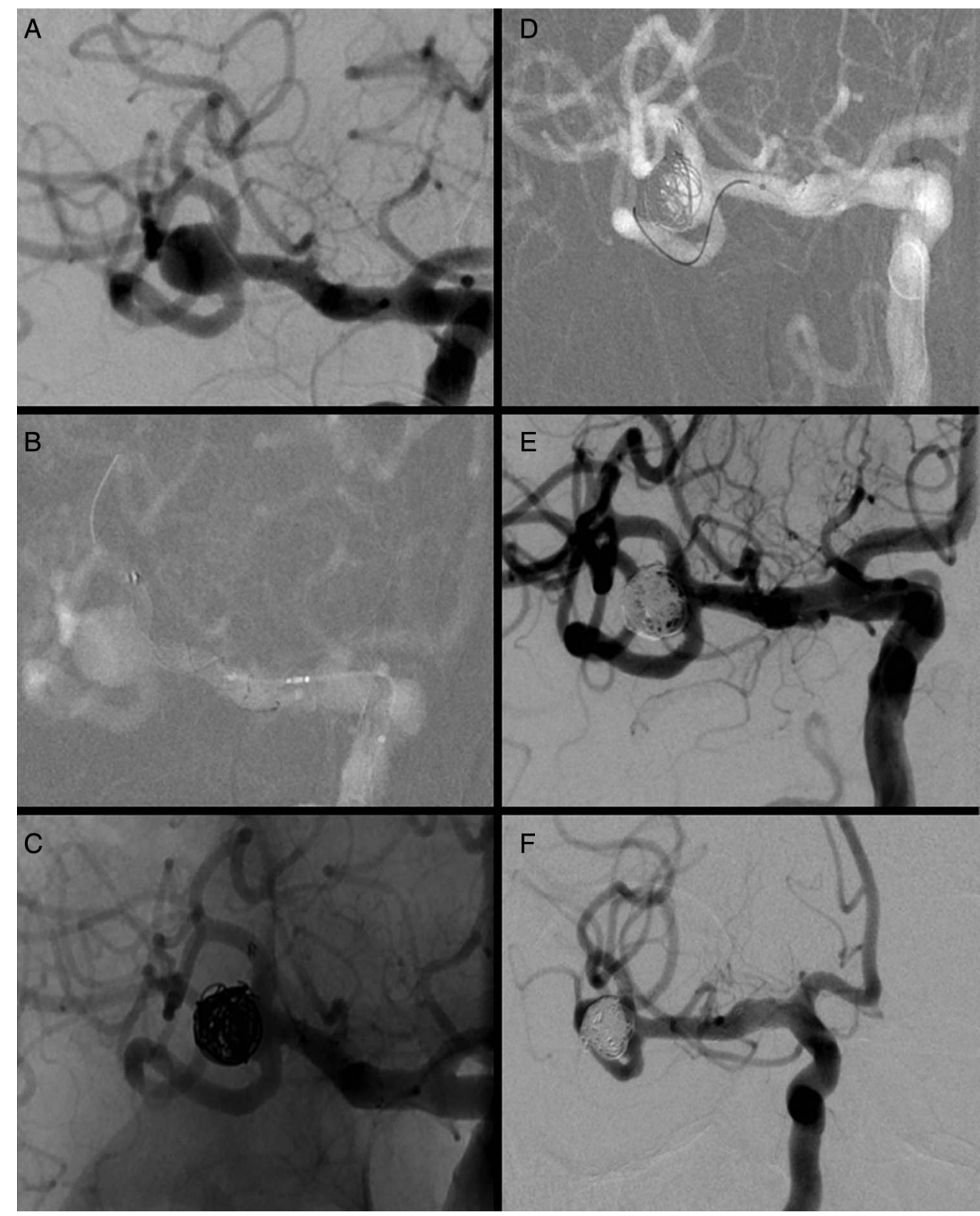

Figure 3 (A) Incidental wide-necked aneurysm in the middle cerebral artery. (B) Deployment of LVIS stent (3/25 mm) in the superior truncus. (C) Coil embolization through the stent struts. (D, E) Deployment of LVIS Jr stent $(2.5 / 4 \mathrm{~mm})$ in the inferior truncus and final coiling. (F) Three-month follow-up showing complete occlusion of the aneurysm.

aneurysm size or site of the aneurysm, making it difficult to distinguish a specific group of aneurysms which might benefit from another endovascular treatment. Additionally, there was only a small difference in the occlusion rates of patients who underwent Y-stenting and those who received a single stent, but the subgroups are too small to address this issue in depth.

Complications occurred in 5/34 cases (15\%) including two cases of thrombus formation after stent placement, so the deployment and implantation can be considered safe although the safety has to be investigated in detail in future trials with more cases. Both cases of thrombus formation were successfully treated with intravenous administration of eptifibatide. The number of thromboembolic complications in our series was comparable to the rates of symptomatic thromboembolic complications ranging from $1.4 \%$ to $8.7 \%$ with either the Neuroform or the Enterprise stent; however, in our series these complications were asymptomatic. In addition, we did not observe any thromboembolic complication in the group of patients who underwent Y-stenting.

\section{CONCLUSION}

The deployment and implantation of the LVIS Jr seems to be safe and effective. Due to the low profile of the LVIS Jr, it can be deployed via a small-lumen microcatheter or dual-lumen balloon which thereby creates the ability to access a more difficult and distal angiographic architecture. Additionally, we observed thrombus formation to be rare (2/34, 5.9\%), which might indicate the potential for a very low number of thromboembolic complications with this device. 
11 Piotin M, Blanc R, Spelle L, et al. Stent-assisted coiling of intracranial aneurysms: clinical and angiographic results in 216 consecutive aneurysms. Stroke 2010;41:110-15.

12 Vendrell JF, Costalat V, Brunel $H$, et al. Stent-assisted coiling of complex middle cerebral artery aneurysms: initial and midterm results. AJNR Am J Neuroradiol 2011;32:259-63.

13 Spiotta AM, Gupta R, Fiorella D, et al. Mid-term results of endovascular coiling of wide-necked aneurysms using double stents in a $Y$ configuration. Neurosurgery 2011;69:421-9.

14 Thorell WE, Chow MM, Woo HH, et al. Y-configured dual intracranial stent-assisted coil embolization for the treatment of wide-necked basilar tip aneurysms. Neurosurgery 2005;56:1035-40.

15 Wanke I, Forsting M. Stents for intracranial wide-necked aneurysms: more than mechanical protections. Neuroradiology 2008;50:991-8.

16 Aenis M, Stancampiano AP, Wakhloo AK, et al. Modeling of flow in a straight stented and nonstented side wall aneurysm model. J Biomech Eng 1997;119:206-12.

17 Mocco J, Snyder KV, Albuquerque FC, et al. Treatment of intracranial aneurysms with the Enterprise stent: a multicenter registry. J Neurosurg 2009;110:35-9.

\section{REFERENCES}

1 Molyneux A, Kerr R, Stratton I, et al. Subarachnoid Aneurysm Trial (ISAT) of neurosurgical clipping versus endovascular coiling in 2143 patients with ruptured intracranial aneurysms: a randomised trial. Lancet 2002;360:1267-74.

2 Lanzino G, Murad MH, d'Urso PI, et al. Coil embolization versus clipping for unruptured intracranial aneurysms: a meta- analysis of prospective controlled published studies. AJNR 2013;34:1764-8.

3 Moret J, Cognard C, Weill A, et al. The "remodeling technique" in the treatment of wide neck intracranial aneurysms. Angiographic results and clinical follow-up in 56 cases. Interv Neuroradiol 1997:3:21-35.

4 Shapiro M, Babb J, Becske T, et al. Safety and efficacy of adjunctive balloon remodeling during endovascular treatment of intracranial aneurysm: a literature review. AJNR Am J Neuroradiol 2008;29:1777-81.

5 Pierot L, Cognard C, Spelle L, et al. Safety and efficacy of balloon remodeling technique during endovascular treatment of intracranial aneurysms: critical review of the literature. AJNR Am J Neuroradiol 2012;33:12-15.

6 Spiotta AM, Bhalla T, Hussain MS, et al. An Analysis of inflation times during balloon-assisted aneurysm coil embolization and ischemic complications. Stroke 2011:42:1051-5.

7 Fiorella D, Albuquerque FC, Han $\mathrm{P}$, et al. Preliminary experience using the Neuroform stent treatment of cerebral aneurysms. Neurosurgery 2004;54:6-17.

8 Benitez RP, Silva MT, Klem J, et al. Endovascular occlusion of wide-necked aneurysms with a new intracranial microstent (Neuroform) and detachable coils. Neurosurgery 2004;54:1359-67; discussion 1368.

9 Fiorella D, Albuquerque FC, Deshmukh VR, et al. Usefulness of the Neuroform stent for the treatment of cerebral aneurysms: results at initial $(3-6 \mathrm{mo})$ follow up. Neurosurgery 2005;56:1191-202.

10 Lee YJ, Kim DJ, Suh SH, et al. Stent-assisted coil embolization of intracranial wide-necked aneurysms. Neuroradiology 2005;47:680-9.

18 Fargen KM, Mocco J, Neal D, et al. A multicenter study of stent-assisted coiling of cerebral aneurysms with a $Y$ configuration. Neurosurgery 2013;73:466-72.

19 Higashida RT, Halbach VV, Dowed CF, et al. Initial clinical experience with a new self-expanding Nitinol stent for the treatment of intracranial cerebral aneurysms: the Cordis Enterprise stent. AJNR Am J Neuroradiol 2005;26:1751-6.

20 Weber W, Bendszus M, Kis B, et al. A new self-expanding nitinol stent (Enterprise) for the treatment of wide-necked intracranial aneurysms: initial clinical and angiographic results in 31 aneurysms. Neuroradiology 2007;49:555-61.

21 Lubicz B, Francois 0 , Levivier $M$, et al. Preliminary experience with the Enterprise stent for endovascular treatment of complex intracranial aneurysms: potential advantages and limiting characteristics. Neurosurgery 2008;62:1063-9.

22 Pumar JM, Blanco M, Vaszuez $F$, et al. Preliminary experience with the Leo self-expanding stent for the treatment of intracranial aneurysms. AJNR Am J Neuroradiol 2005;26:2573-7.

23 Juszkat R, Nowak S, Smol S, et al. Leo stent for endovascular treatment of broad necked and fusiform intracranial aneurysms. Intervent Neuroradiol 2007;13:255-69.

24 Kis B, Weber W, Berlit $P$, et al. Elective treatment of saccular and broad-necked intracranial aneurysms using a closed-cell Nitinol stent (Leo). Neurosurgery 2006;58:443-50

25 Turner RD, Turk A, Chaudry MI. Low-profile visible intraluminal support device: immediate outcome of the first three US cases. J Neurointerv Surg 2013;5:157-60.

26 Spiotta AM, Miranpuri A, Chaudry MI, et al. Combined balloon stent technique with the Scepter C balloon and low-profile visualized intraluminal stent for the treatment of intracranial aneurysms. J Neurointerv Surg 2013;5(Suppl 3): iii79-82.

27 Kadkhodayan Y, Rhodes N, Blackburn S, et al. Comparison of Enterprise with Neuroform stent-assisted coiling of intracranial aneurysms. AJR Am J Roentgenol 2013;200:872-8. 